\title{
Grandeur et décadence des femmes peintres entre la fin de la monarchie et la première moitié du $\mathrm{XIX}^{\mathrm{e}}$ siècle
}

The rise and fall of women painters, from end of the monarchy to the first half of the nineteenth century

\section{Vera de Ladoucette}

\section{OpenEdition}

Journals

Édition électronique

URL : https://journals.openedition.org/cel/15849

DOI : $10.4000 /$ cel. 15849

ISSN : 2262-208X

\section{Éditeur}

École du Louvre

\section{Référence électronique}

Vera de Ladoucette, «Grandeur et décadence des femmes peintres entre la fin de la monarchie et la première moitié du XIXe siècle », Les Cahiers de l'École du Louvre [En ligne], 16 | 2021, mis en ligne le 04 juin 2021, consulté le 30 juillet 2021. URL : http://journals.openedition.org/cel/15849 ; DOI : https:// doi.org/10.4000/cel.15849

Ce document a été généré automatiquement le 30 juillet 2021

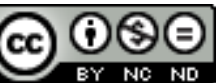

Les Cahiers de l'École du Louvre sont mis à disposition selon les termes de la licence Creative Commons Attribution - Pas d'Utilisation Commerciale - Pas de Modification 4.0 International. 


\section{Grandeur et décadence des femmes peintres entre la fin de la monarchie et la première moitié du XIX ${ }^{\mathrm{e}}$ siècle}

The rise and fall of women painters, from end of the monarchy to the first half of the nineteenth century

Vera de Ladoucette

1 Si les représentations du peintre par lui-même se multiplient à partir de la Renaissance, le terme « autoportrait » n'apparaît que dans la seconde moitié du xIx siècle en France et se généralise très lentement ${ }^{1}$. Il faut attendre la fin $\mathrm{du} \mathrm{xx}^{\mathrm{e}}$ siècle pour voir des historiens de l'art, notamment Pascal Bonafoux ${ }^{2}$ et Omar Calabrese ${ }^{3}$, consacrer des ouvrages entiers à la question de l'autoportrait, des intellectuels, comme Philippe Lejeune, s'y intéresser également ${ }^{4}$, et les musées y consacrer de nombreuses expositions ${ }^{5}$. Parallèlement, la montée en puissance des femmes artistes ${ }^{6}$ dans le dernier quart du XVIII ${ }^{e}$ siècle a fait l'objet d'études, notamment par Marie-Jo Bonnet ou Séverine Sofio sur le plan sociologique et politique ${ }^{7}$, alors que Frances Borzello a dressé une histoire de l'autoportrait féminin's.

2 En 2021, le musée du Luxembourg propose une exposition qui fait date, Peintres femmes, Naissance d'un combat, 1780-1830'. L'objectif de Martine Lacas, la commissaire de l'exposition, n'est pas de présenter un parcours linéaire de " peintres femmes ${ }^{10}$, fondé sur l'hypothèse d'un progrès inéluctable de la condition féminine dans le monde de l'art, mais de faire découvrir, au-delà des rares femmes artistes incontournables, une trentaine de peintres "méconnues voire inconnues du grand public ${ }^{11}$ », qui, pour la plupart, ont pratiqué l'autoportrait. Ainsi, cette exposition permet d'admirer l'Autoportrait de Rosalie Filleul de Besne ${ }^{12}$, qui, n'étant pas académicienne, a dû se contenter de briller à l'Académie de Saint-Luc ${ }^{13}$, comme l'Autoportrait de Julie Duvidal de Montferrier ${ }^{14}$, présenté, lui, au Salon de 1819.

3 Néanmoins, même si l'exposition du Luxembourg consacre une large place aux autoportraits des femmes, aucune recherche n'a exploré de manière approfondie la 
manière dont la représentation des femmes par elles-mêmes a évolué sur trois décennies - de la fin de la monarchie au début de l'Empire -, en marquant un réel renversement de la tendance avec l'effacement progressif de la revendication de la femme peintre en tant qu'artiste.

4 La méthode statistique nous a paru être une voie complémentaire utile pour explorer l'évolution de la place des femmes à cette époque charnière. Nous suivons ainsi l'exemple donné dans des domaines similaires par des historiens d'art comme Udolpho van der Sandt ou Pascal Griener et Paul-André Jaccard. Ainsi, l'outil statistique a été mis à profit par van der Sandt pour nous éclairer sur la fréquentation du Salon, notamment à travers la vente des livrets ${ }^{15}$, alors que Griener et Jaccard ont pu documenter l'activité des quelque quatre cents artistes suisses formés à Paris grâce à l'exploitation systématique des Archives nationales ${ }^{16}$.

5 L'évolution de la participation au Salon des femmes artistes peut être résumée par l'étude de deux tableaux, peints à vingt-trois ans d'intervalle; cette étude nous permettra de remonter le temps et d'explorer la place de l'autoportrait, véritable passeport pour le Salon pour les femmes à l'aube de la Révolution, jusqu'à ce qu'un effacement progressif de ces mêmes femmes peintres soit mis en évidence par la transformation radicale de la manière dont elles se représentent ${ }^{17}$. Pour ce faire, nous nous appuierons sur une étude statistique et qualitative, fondée tant sur le dépouillement des livrets du Salon jusqu'en $1848^{18}$, que sur l'analyse des œuvres présentées au Salon.

\section{Le constat : un retour en arrière spectaculaire pour les femmes artistes}

6 À vingt-trois ans de distance, deux représentations d'Adélaïde Labille-Guiard ${ }^{19}$, une des femmes peintres les plus en vue à la fin $d u$ xvIII ${ }^{e}$ siècle, résument parfaitement l'évolution de la condition des artistes de sexe féminin dans la première moitié du $\mathrm{XIX}^{\mathrm{e}}$ siècle $^{20}$.

7 Lorsque Labille-Guiard présente Autoportrait avec deux élèves ${ }^{21}$ (fig. 1), au Salon de 1785, l'éloge est unanime :

«Son tableau, qui frappe le plus et le sujet de l'admiration générale, est un tableau historié, où elle s'est figurée elle-même en pied, occupée à peindre, avec deux de ses élèves derrière elle, considérant l'ouvrage de leur maîtresse et épiant, pour ainsi dire, le moment de surprendre le secret d'un si rare talent. Unité d'action, plan net, intention bien sentie, beaux choix de nature, attitudes variées, vraies et naturelles, grande intelligence du clair-obscur, tons sûrs, coloris harmonieux, accord de la grâce et de la vigueur, tout ce qu'on peut désirer se trouve réuni dans cette composition savante et digne des plus grands maîtres ${ }^{22}$.» 
Figure 1.

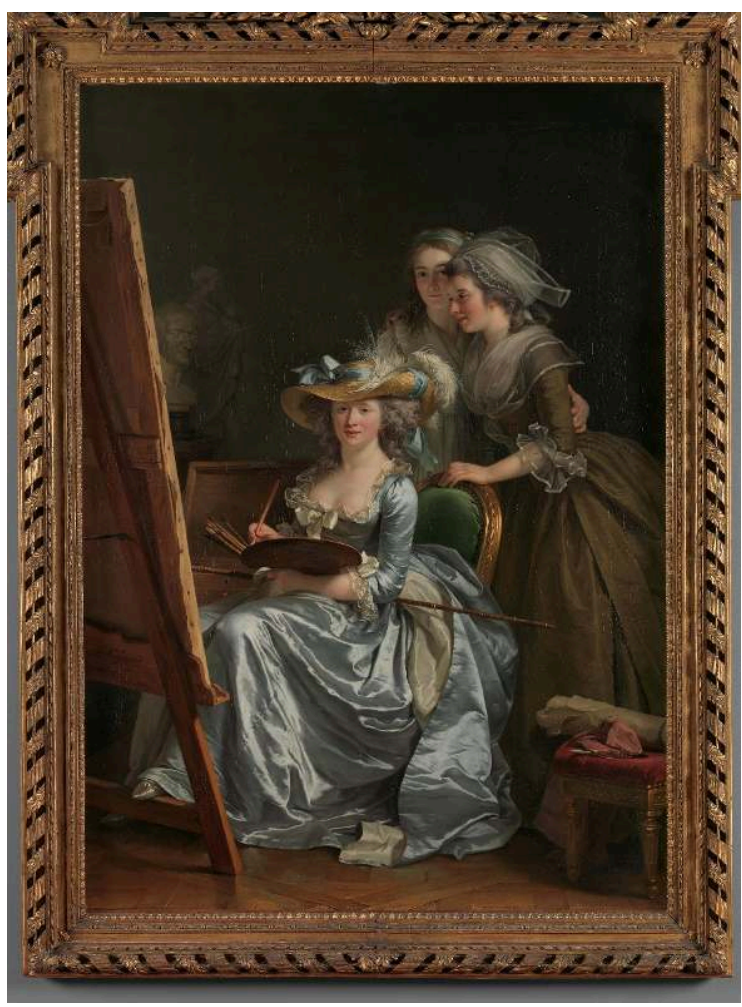

A. Labille Guiard, Autoportrait avec deux élèves, 1785, huile sur toile, H. 2,10 m ; L 1,51 m, New York, Metropolitan museum, 53.225.5.

Pour Labille-Guiard, dont certains ${ }^{23}$ avaient laissé entendre que ses tableaux étaient l'œuvre de François-André Vincent ${ }^{24}$, son professeur et futur époux, l'objectif est double : rappeler, en se montrant au travail, qu'elle est bien l'auteur de ses tableaux ${ }^{25}$, mais aussi, par une mise en abyme, mettre en scène l'admiration qu'elle doit susciter ${ }^{26}$. Elle se représente en effet avec toutes les armes de la séduction d'une femme, mais également comme une artiste reconnue au point d'enseigner la peinture à deux de ses élèves.

9 La deuxième représentation n'est pas due au pinceau d'Adélaïde Labille-Guiard, bien qu'elle en soit le sujet principal, mais à celui de son élève préférée, Marie-Gabrielle Capet, qui figure à l'arrière-plan de l'Autoportrait avec deux élèves. Au Salon de 1808, elle rend hommage à son professeur avec le Tableau représentant feue Madame Vincent, élève de son mari (fig. 2). Le titre est explicite. L'image l'est encore plus. Labille-Guiard n'est plus la femme séduisante et l'artiste triomphante du tableau présenté au Salon de 1785. Vêtue simplement, elle redevient l'élève de Vincent, qui, penché sur elle, pointe sa main sur la toile, alors qu'elle fait le portrait du peintre et sénateur Joseph-Marie Vien. Quant à Marie-Gabrielle Capet, humblement assise aux pieds de son ancienne enseignante, elle charge sa palette. 
Figure 2.

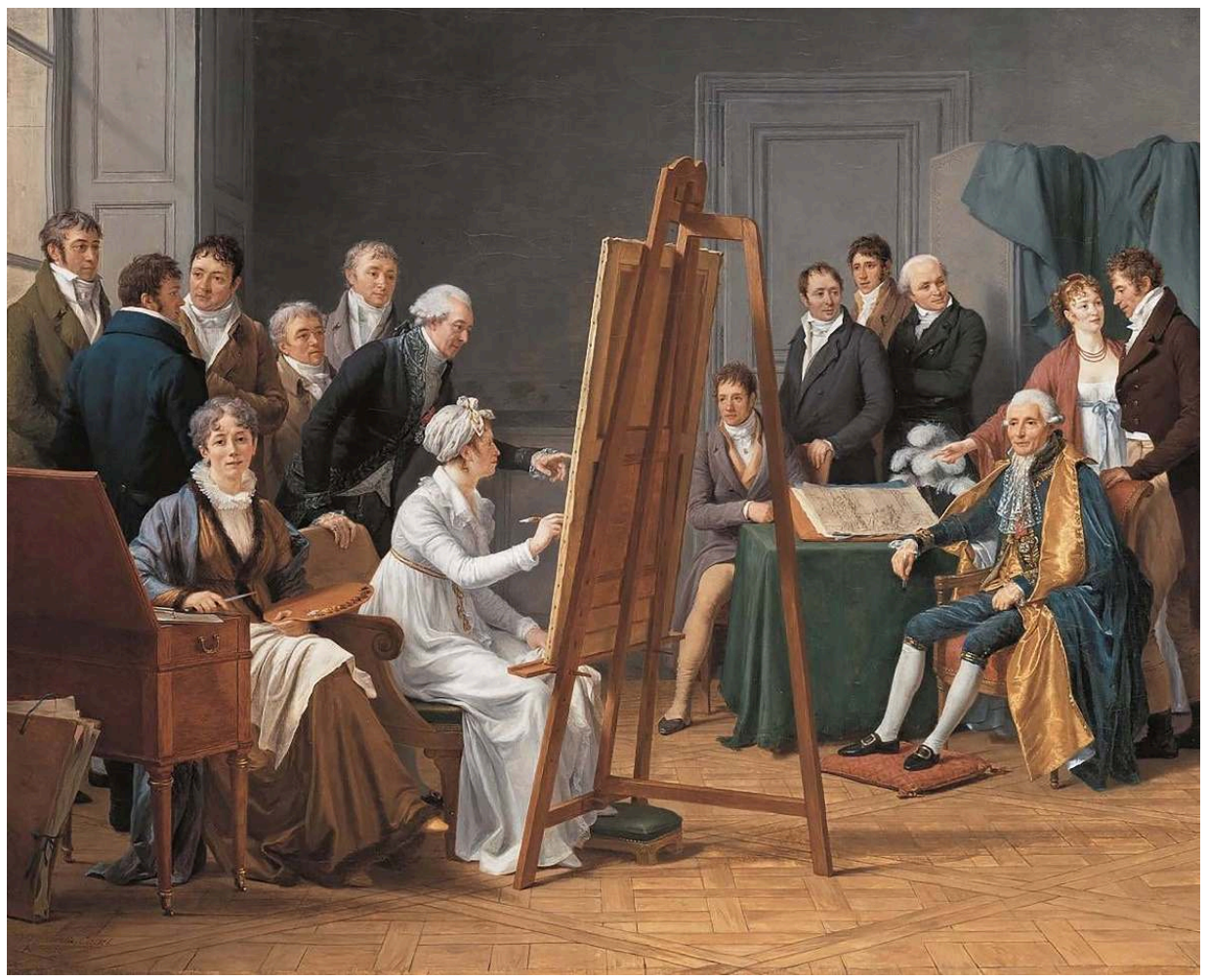

M.-G. Capet, Tableau représentant feue Madame Vincent, élève de son mari, 1808, huile sur toile, H. 0,69 m ; L. 0,83 m, Munich, Neue Pinakothek, FV 9.

10 Un retour en arrière s'impose pour comprendre que le décalage entre ces deux représentations de la même femme peintre est lié à la modification du contexte artistique, mais aussi social, à la charnière entre la Révolution et l'Empire.

\section{Les années 1780, une décennie clémente pour les femmes dans le monde artistique}

Au XVIII ${ }^{e}$ siècle, le cercle des arts est pratiquement régi par un double système, partagé entre un système corporatif - avec l'Académie de Saint-Luc ${ }^{27}$-, et l'Académie Royale de Peinture et de Sculpture ${ }^{28}$, créée en 1648 par le cardinal Mazarin. Il y a un véritable fossé entre le monde des artisans, c'est-à-dire des «travailleurs manuels ", affiliés à l'Académie de Saint-Luc qui protège leurs droits, et celui des peintres admis à l'Académie, qui constituent une véritable aristocratie culturelle et se différencient entre eux en fonction de la hiérarchie des genres, codifiée par André Félibien en $1667^{29}$.

La réussite ultime pour une femme passe, jusqu'à la Révolution, par l'admission à l'Académie. En effet, appartenir à l'Académie est une question de prestige, lié à la qualité de membre de cette Institution, car, renforçant le statut des artistes, elle établit une frontière nette entre ceux-ci et les artisans. C'est également une nécessité, car ses membres constituent une caste privilégiée, qui, seule, a accès au Salon. Or, l'admission des femmes est limitée, voire impossible, pendant une large part du XviII ${ }^{\mathrm{e}}$ siècle. La première femme est admise à l'Académie le 14 avril 1663 lorsque : 
«... [suivant] l'intention du Roi, qui est d'épandre sa grâce sur tous ceux qui y excellent dans l'art de la Peinture et de la Sculpture, d'en faire part à ceux qui en seront jugés dignes, sans avoir égard à la différence du sexe, Monsieur Le Brun ayant présenté un tableau de fleurs par Mademoiselle Girardon ${ }^{30}$, toute la Compagnie, touchée de l'estime dudit ouvrage et connaissant le mérite de cette demoiselle, a décidé de lui donner le titre d'Académicienne ${ }^{31}$. "

13 portraitiste. Cette parenthèse est brève. Les femmes sont exclues officiellement de l'Académie le 25 septembre 1706 :

«l'Académie, étant assemblée à l'ordinaire, sur ce qui lui avait été proposé que plusieurs Demoiselles qui se sont appliquées à la Peinture avaient le dessein de se présenter pour être reçues Académiciennes, la Compagnie après avoir fait une sérieuse réflexion, pour prévenir ces présentations a résolu que dorénavant on ne recevrait aucune Demoiselle en qualité d'Académicienne, et que cette résolution servirait de règlement général ${ }^{33}$.»

Le règlement de 1706 n'est qu'imparfaitement appliqué : des exceptions sont faites pour quelques femmes peintres, en raison de leur origine étrangère $e^{34}$, mais pendant cinquante-trois ans les femmes sont, de facto, exclues du Salon. Elles parviennent cependant à contourner partiellement l'obstacle grâce à des expositions parallèles.

À la dissolution de l'Académie de Saint-Luc, les artistes qui n'en sont pas membres trouvent refuge au Salon de la Correspondance, organisé tous les ans entre 1779 et 1787 par Mammès-Claude Pahin de la Blancherie ${ }^{35}$. Ce nouvel espace permet aux femmes de commencer à s'affirmer en tant qu'artistes. En termes relatifs, la croissance parait impressionnante: le pourcentage des artistes femmes par rapport au nombre total d'exposants double, passant de $8 \%$ en 1779 à $15 \%$ en 1787. En chiffres absolus, c'est moins spectaculaire. Émile Bellier de la Chavignerie a dressé la liste des artistes ayant exposé au Salon de la Correspondance. Sur deux-cent-vingt-cinq participants, dix-sept sont des femmes peintres, et on relève le nom d'une seule femme sculptrice, Julie Charpentier (1770-1845), élève de Pajou, qui présente son Autoportrait en 1793.

Par ailleurs, les femmes artistes sont de plus en plus actives à l'Exposition de la Jeunesse qui se tient en plein air, Place Dauphine et sur le Pont-Neuf, depuis la seconde moitié du XVII siècle, le jour de la Fête-Dieu. Thomas Crow souligne que de nombreux futurs Académiciens y connurent leurs premières heures de gloire ${ }^{36}$. Certes, aucun livret n'en fut publié et sa durée était très limitée - une matinée -mais l'Exposition de la Jeunesse bénéficiait d'articles réguliers, quoique succincts, dans la presse. Or l'examen des comptes rendus de cette manifestation confirme la lente montée en puissance des femmes à partir des années 1760. L'Avant-Coureur mentionne la présence de deux femmes en 1760, trois en 1761, sept en 1767. Les recensions du Mercure de France évoquent les œuvres de neuf femmes et de six hommes en 1780 ; cinq femmes pour cinq hommes en 1784 ; sept femmes pour sept hommes en 1785. La parité, notion qui n'existe pas encore, est ainsi atteinte ${ }^{37}$ !

17 En 1754, l'interdiction pour les femmes d'appartenir à l'Académie est levée en faveur de Marie-Thérèse Reboul (1728-1805), épouse de l'Académicien Joseph-Marie Vien. L'étape suivante a lieu en $1770^{38}$ : Anne Vallayer ${ }^{39}$ est agréée et reçue le 28 juillet, après avoir présenté deux tableaux, Les Attributs de la peinture, de la sculpture et de l'architecture et son pendant Les Instruments de musique. Elle est alors la seule femme à être admise sans être épouse ou fille d'académicien, ni officiellement recommandée ${ }^{40}$. Deux Académiciennes, admises en moins d'un mois! La Compagnie s'effraie de sa propre audace, et, un mois 
plus tard, sous l'impulsion du marquis de Marigny, directeur des Bâtiments du Roi (et frère de Madame de Pompadour), édicte un règlement afin de limiter à quatre le nombre de membres femmes. Ce texte du 28 septembre 1770 est clair, voire abrupt :

«L'Académie ayant considéré que, quoiqu'Elle se fasse un plaisir d'encourager le talent dans les femmes en en admettant quelques-unes dans son Corps, néanmoins ces admissions, étrangères en quelque façon à sa constitution, ne doivent pas être trop multipliées; Elle a arrêté qu'Elle n'en recevrait point au-delà du nombre de quatre [...]. L'Académie au reste ne prétend pas s'engager à remplir toujours le nombre de quatre, se réservant de ne le faire qu'autant qu'Elle s'y trouvera déterminée par des talens ${ }^{41}[$ sic $]$ véritablement distingués ${ }^{42}$. "

$18 \mathrm{Au}$ total, quinze femmes auront été membres de l'Académie ${ }^{43}$. C'est peu par rapport à cinq-cent-trente-neuf hommes. Ajoutons cependant qu'un autre parcours est possible, même si les réussites constituent des exceptions. L'exemple le plus emblématique est celui de Marguerite Gérard ${ }^{44}$, qui n'est pas membre de l'Académie et ne participe pas au Salon jusqu'en 1798, mais entretient des liens étroits avec collectionneurs et marchands ${ }^{45}$. Si le Louvre a reconnu tardivement Marguerite Gérard, elle eut beaucoup de succès dans le premier tiers du XIX ${ }^{e}$ siècle et ses tableaux sont exposés depuis longtemps en Russie, comme en Allemagne. L'entrée au Louvre en 2019 de son tableau L'Élève intéressante ${ }^{46}$ représente, à ce jour, la dernière acquisition d'un autoportrait par le musée. L'œuvre est à la jonction entre plusieurs catégories, étant à la fois un portrait de jeune artiste, l'élève, une réflexion sur la formation des femmes artistes au $\mathrm{xvIII}^{\mathrm{e}}$ siècle dans le cadre d'atelier familiaux, une double scène d'atelier (celui de l'élève et celui de Marguerite Gérard), ainsi qu'un autoportrait in assistenza; le tout méritant notamment notre attention par la prouesse technique qui nous permet de voir l'autre partie de l'atelier reflétée dans la surface polie de la sphère métallique.

\section{L'autoportrait, un moyen pour les femmes de s'approprier le Salon}

19 Au Salon de la Correspondance comme à l'Exposition de la Jeunesse, les femmes avaient déjà commencé à se faire remarquer par leurs autoportraits. Un an après avoir exposé chacune un autoportrait au Salon de la Correspondance, les autoportraits de LabilleGuiard et Vigée Le Brun suscitent l'intérêt à l'Exposition de la Jeunesse de 1782. C'est dans ce cadre que cette dernière présente l'Autoportrait au chapeau de Paille ${ }^{47}$. Ce n'est pas son premier autoportrait; elle en a déjà réalisé sept et en peindra encore une quinzaine $^{48}$. À l'Exposition de la Jeunesse de 1786, les autoportraits des jeunes élèves d'Adélaïde Labille-Guiard frappent les esprits :

\footnotetext{
«J'entre jeudi 22 juin 1786 dans la place Dauphine et mon premier soin est de faire le tour de la galerie; j'aperçois un grand nombre de portraits, je les examine, et j'entends répéter autour de moi à plusieurs reprises "c'est le portrait de Melle... peint par elle-même". Un instant après, j'entends dire et répéter "on peut juger des ressemblances, car les originaux sont ici. Regardés aux fenêtres, vous les verrés" [sic]. Je lève les yeux et vois en effet une demie douzaine de balcons chargés de jeunes personnes, parées les unes de leurs charmes naturels, les autres de tous les embellissements de la toilette ${ }^{49}$."
}

Enfin admises à l'Académie en mai 1783, Vigée Le Brun et Labille-Guiard ne respectent pas la discrétion dont avaient fait preuve, jusqu'alors, leurs aînées. À peine élues, ces deux Académiciennes font évènement en présentant de nouveaux autoportraits. Au 
Salon de 1783, le nom de Vigée Le Brun est sur toutes les lèvres. Dès le début de sa recension du Salon de 1783, Mouffle d'Angerville donne le ton :

«Lorsque quelqu'un annonce qu'il arrive du Salon, on lui demande d'abord, avezvous vu Madame Le Brun? Que pensez-vous de Madame Le Brun? Et en même temps on lui suggère sa réponse : N'est-il pas vrai que c'est une femme étonnante, que madame Le Brun? tel est son nom, Monsieur, de la femme que j'ai en vue, devenue si célèbre en peu de temps; car elle n'est que depuis quelques mois reçue académicienne d'emblée, suivant le privilège de son sexe, et dans une des quatre places qui lui sont uniquement et spécialement affectées ${ }^{50}$. »

21 Le critique loue également Labille-Guiard et admire plus spécialement son autoportrait : « son corps est penché en avant et dans cette attitude d'abandon où l'âme est toute entière occupée de son objet. Sa tête vigoureuse annonce les conceptions fortes dont elle est pleine; et son vêtement simple et pittoresque atteste et son talent et sa modestie $e^{51}$.»

Les deux artistes ne se contentent pas de se représenter, elles se revendiquent en tant que peintres, tenant palette et pinceau ${ }^{52}$. L'autoportrait «à la peinture » est un moyen de s'affirmer en tant qu'artistes à part entière, de s'approprier le Salon. Une fois cette étape franchie, sur deux décennies - de 1783 à l'Empire -, l'autoportrait redevenait pour les femmes un moyen d'expression parmi d'autres.

\section{Émancipation des femmes « dans » et « par » l'art}

Au XVIII ${ }^{e}$ siècle l'autoportrait « à la peinture » devient pour les femmes le véhicule d'une véritable revendication, artistique mais aussi, parfois, politique. En paraphrasant Joanna Woods-Warden à propos du peintre de la Renaissance, on peut affirmer que le développement de l'autoportrait est une manifestation du combat des femmes pour se faire accepter, à l'égal des hommes, en tant qu'artiste, et non artisan ${ }^{53}$.

Au tournant de la Révolution, on constate un double phénomène, d'une part l'explosion du nombre de femmes participant au Salon, d'autre part une surreprésentation des autoportraits dans leur production.

En 1791, la barrière de l'Académie saute: "Tous les Artistes Français et Étrangers, Membres ou non de l'Académie ${ }^{54} »$ peuvent exposer au Salon. On assiste à une véritable inflation d'exposants, partant d'œuvres. Le nombre d'artistes est multiplié par quatre, le nombre de femmes peintres par dix. On passe de trois femmes qui participent au Salon en 1783 à 21 en 1791 et 34 en 1799. La croissance continue sous l'Empire, avec une première pointe à 77 femmes en 1812 ; la tendance se prolonge sous la Restauration et la monarchie de Juillet, et culmine, pour la période considérée ici, à 295 exposantes en $1848^{55}$, sous la Seconde République.

En termes relatifs, le tableau est plus nuancé. Les artistes femmes représentent 3,8 \% des exposants pendant les trois premiers Salons, avant d'être totalement absentes de cette manifestation pendant cinquante-trois ans. La part des femmes oscille entre 1,5\% et $4,2 \%$ de 1757 à 1789 , monte à $6,3 \%$ dès le premier Salon de la Révolution et croît jusqu'à une première pointe de $15,4 \%$ en 1802 . Elle se stabilisera ensuite autour de $12 \%$ pendant la Restauration et les premières années de la monarchie de Juillet, avant de connaître un deuxième pic à plus de $16 \%$ en 1836-1837. La moyenne revient ensuite à $13 \%$ pendant la dernière décennie de la monarchie de Juillet (fig. 3 ). 
Figure 3.

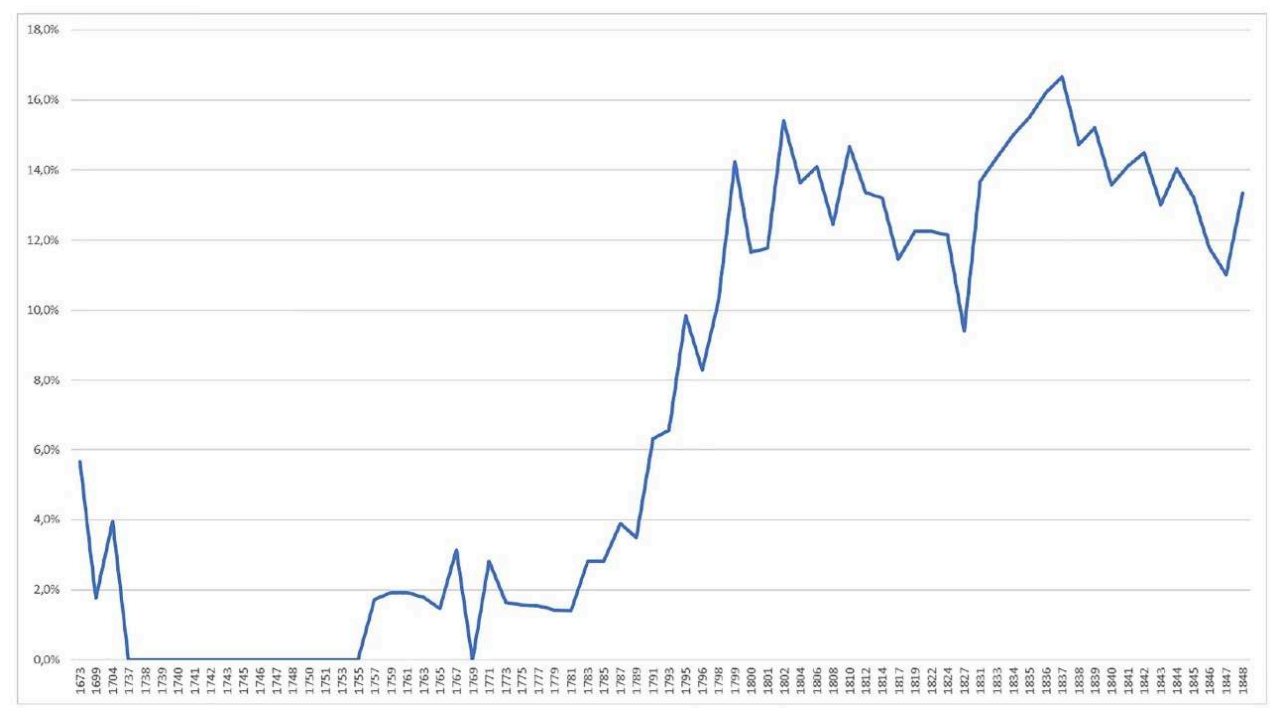

Vera de Ladoucette, Part des femmes par rapport au nombre total d'exposants au Salon.

(c) Ladoucette

Parallèlement, il convient de souligner, tout d'abord d'un point de vue quantitatif, le rôle des femmes dans la production des autoportraits exposés au Salon. Quand il y a un seul autoportrait, et que l'auteur en est une femme, comme dans les années 1785-1789, parler de pourcentage paraît dérisoire, mais les deux décennies suivantes sont plus significatives, car les autoportraits de femmes représentent en moyenne un tiers des autoportraits exposés au Salon, avec un record en 1793 avec plus de la moitié des 14 autoportraits exposés, comme le montre le tableau en annexe 2 . Dès la chute de l'Empire, la part des femmes dans le nombre d'autoportraits tombe à $12,5 \%$ en 1812 ; il s'établit en moyenne à moins de $18 \%$ pendant les décennies suivantes, mais ce chiffre masque des variations considérables: ainsi, constate-t-on l'absence d'autoportraits féminins aux Salons de 1835 et 1839, alors qu'en 1822 comme en 1831 les autoportraits de femmes représentent un tiers de l'ensemble des autoportraits (fig. 4). 
Figure 4.

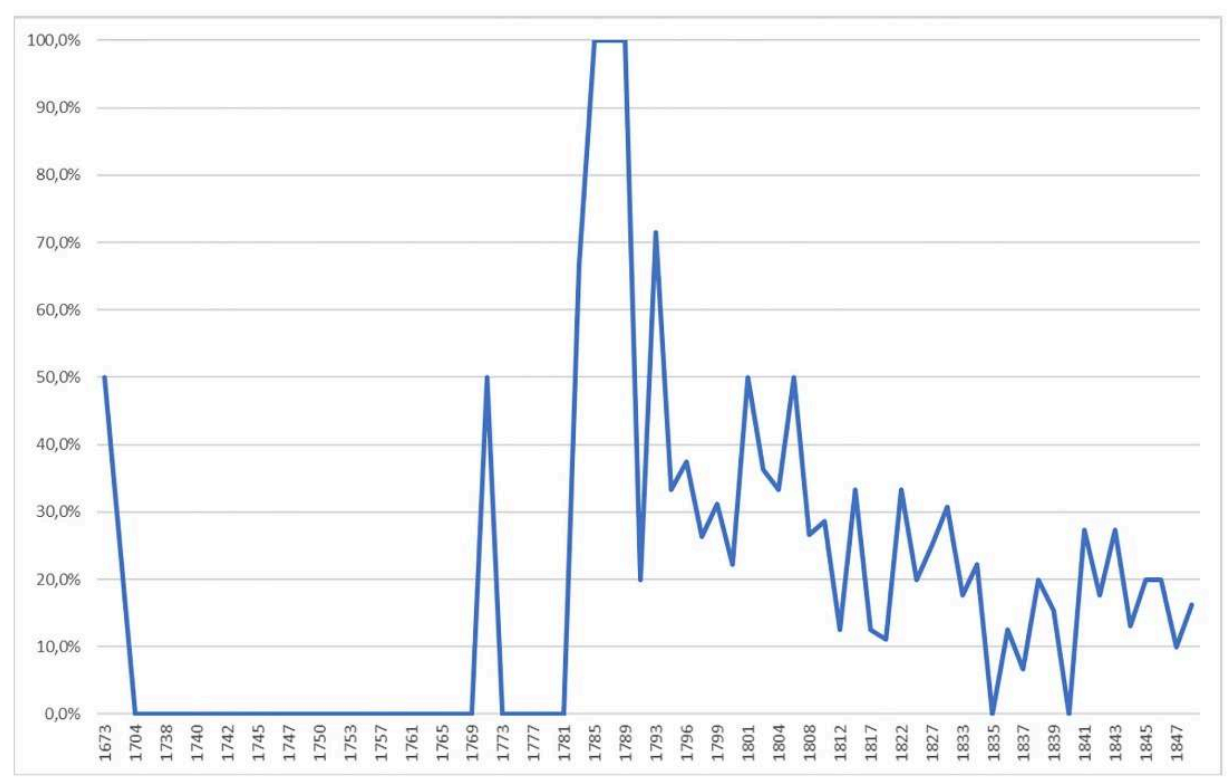

Vera de Ladoucette, Part des autoportraits de femmes par rapport au nombre total d'autoportraits au Salon.

(C) Ladoucette

Comme toujours, rester sur le plan quantitatif serait cependant réducteur. L'appropriation du Salon par les femmes passe non seulement par les autoportraits, mais surtout par la revendication de leur statut de femmes artistes. En se représentant en tant que peintres, elles témoignent de leur volonté d'émancipation ${ }^{56}$. Il s'agit essentiellement d'une émancipation " par l'art ». C'est en effet l'art qui met ces femmes au premier plan de la vie publique.

Elles se représentent en train de peindre ou personnifiant la peinture. On compte deux tableaux sur ce thème en 1791, neuf en 1793, un en 1795, cinq en 1796, deux en 1798 , entre un et trois par Salon pendant l'Empire; elles se peignent également en train de donner des leçons de peinture: l'œuvre emblématique étant L'Autoportrait avec deux élèves de Labille-Guiard.

Au-delà même de leur représentation en tant qu'artiste, les femmes peintres affichent leur ambition d'être considérées en tant qu'égales de leurs homologues masculins; dans cet objectif, elles multiplient les références aux arts dans leurs autoportraits, en citant soit des grands maîtres, soit encore des artistes contemporains.

Chéron, la première, se représente tenant un dessin, sur lequel on distingue l'esquisse d'un portrait de femme. Labille-Guiard place des bustes néoclassiques en arrière-plan de l'Autoportrait avec deux élèves ${ }^{57}$. Vigée Le Brun revendique ses références; elle raconte comment elle s'est inspirée de Rubens pour l'Autoportrait au chapeau de paille (fig. 5) :

«À Anvers, je trouvai chez un particulier le fameux chapeau de paille [...]. Ce tableau me ravit et m'inspira au point que je fis mon portrait à Bruxelles en cherchant le même effet. Je me peignis portant sur la tête un chapeau de paille, une plume et une guirlande de fleurs des champs, et tenant ma palette à la main ${ }^{58}$. » 
Figure 5.

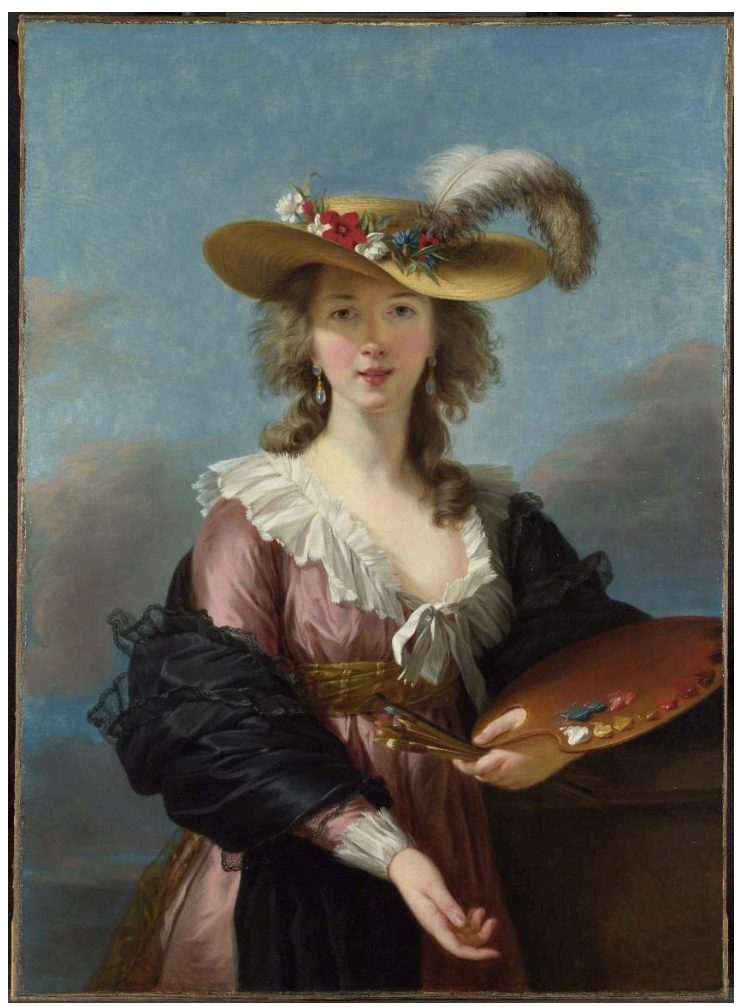

E. Vigée-Le Brun, Autoportrait au chapeau de paille, 1782, huile sur toile, H. 0,97 m ; L 0,70 m, Londres, National Gallery, NG 1653.

(c) The National Gallery, Londres, Dist. RMN-Grand Palais / National Gallery Photographic Department

En 1787, elle se tourne vers l'Italie pour l'Autoportrait avec sa fille Julie, aussi appelé La Tendresse maternelle. Les critiques ne s'y trompent pas et identifient sans peine sa source d'inspiration : La Vierge à la chaise de Raphaël. Dans la Lettre d'un étranger sur le Salon de 1787 le comte Potocki ${ }^{59}$ affirme que ce tableau «tient beaucoup pour sa composition de la fameuse "Madonne de la Sedia" ${ }^{60}$. » Douze ans plus tard, Constance Mayer ${ }^{61}$ se réfère au même maître en présentant son Portrait en pied d'un père et de sa fille. Sur cette peinture, le père indique à sa fille le buste de Raphaël en l'invitant à prendre pour modèle ce peintre célèbre ${ }^{62}$ (fig. 4).

D'autres femmes préfèrent se confronter à leurs homologues contemporains. Suzanne Roslin $^{63}$, dans un tableau qui n'est pas présenté au Salon, se peint faisant une copie de l'autoportrait de Quentin de La Tour en Homme qui rit, tandis que Marie-Guilhelmine Benoist $^{64}$ se réfère au Bélisaire de David. Non seulement la jeune artiste s'approprie le tableau d'un contemporain, mais en outre sa toile fait date puisqu'elle rompt avec le style roccoco. En effet, avec Bélisaire demandant l'aumône, quatre ans avant Le Serment des Horaces, David présentait au Salon de 1781 sa première toile néoclassique, devenant ainsi le chef-de-file de ce nouveau mouvement. En copiant Bélisaire, Benoist revendiquait donc son inscription au sein du courant.

La démarche de ces femmes peintres est originale. Certes, des hommes se représentent dans leur atelier, mais quand on examine leurs autoportraits présentés au Salon, point de référence aux Anciens, et encore moins à leurs contemporains. Les autoportraits du dernier quart du XVIII siècle sont convenus, comme les miniatures de Jean-Baptiste Jacques Augustin ${ }^{65}$, introspectifs, comme les pastels de Chardin ${ }^{66}$, ironiques, tels les 
tableaux de Ducreux ${ }^{67}$, alors que les portraits de peintre dans l'atelier montrent le plus souvent l'artiste en majesté, comme Van Loo, conseillé par sa sœur, peignant le portrait de son père ${ }^{68}$.

Pour une infime minorité de ces femmes, la revendication est politique : l'exigence d'égalité avec leurs confrères masculins dans le domaine artistique. Labille-Guiard, conjointement avec le graveur Miger ${ }^{69}$ et Moreau le Jeune ${ }^{70}$, prend la tête de ce mouvement ${ }^{71}$. En 1791, elle propose à l'Académie deux motions : la première vise à supprimer le décret limitant le nombre de femmes, et la seconde l'admission des femmes comme " conseillères ", dans la mesure où jusqu'alors celles-ci ne pouvaient ni enseigner ni occuper des fonctions administratives ${ }^{72}$. Ces motions sont votées, non sans difficulté, le 23 septembre 1790, mais les officiers qui s'opposent à ces réformes, font sécession 48 heures après. Ce vote ne se transcrira jamais dans les faits, d'autant que l'Académie est dissoute, à l'initiative de David ${ }^{73}$, le 8 août 1792.

Pour les artistes femmes, le bilan de la Révolution est mitigé. Certaines portes s'ouvrent, mais d'autres se ferment. En effet, en 1793, la Société populaire et républicaine des Arts (succédané de l'Académie) exclut les femmes de ses réunions, au prétexte qu'elles sont « en tout point différentes des hommes ${ }^{74}$.» Évincées de ce nouvel aréopage artistique, les femmes continueront cependant d'exposer au Salon.

\section{Le recul du rôle des femmes peintres au début du $\mathrm{XIX}^{\mathrm{e}}$ siècle se traduit par l'abandon progressif de l'autoportrait}

37 Nous avons vu qu'avec les années, le nombre de femmes participant au Salon ne cesse de croître, alors que le nombre d'autoportraits de femmes se stabilise en valeur absolue, mais décline fortement par rapport au nombre d'œuvres présentées. L'autoportrait leur a servi de porte d'entrée, il leur a permis de s'approprier le Salon. Une fois établies dans leurs droits, les artistes femmes n'ont plus besoin de démontrer leur légitimité. Il semble qu'elles peuvent se permettre d'aborder tous les genres, y compris, si telle est leur envie, l'autoportrait.

Il convient pourtant de nuancer ce propos. Certes, la fin de la Révolution ne met pas un coup d'arrêt brutal aux ambitions artistiques des femmes, mais le phénomène est plus graduel, plus pernicieux également. Le contexte a changé. La régression de la condition féminine, amorcée pendant la Révolution, est accentuée par le Code civil de 1804, qui déclare la femme incapable juridiquement ${ }^{75}$; elle passe de l'autorité de son père à celle de son mari, le chef de famille, auquel elle doit « obéissance ${ }^{76}$ ». Les valeurs bourgeoises enferment la femme, dont la seule vocation est désormais la maternité, ce qui conduit Madame de Staël à regretter la liberté de l'aristocratie des Lumières ${ }^{77}$. La bourgeoisie triomphante de la monarchie de Juillet conforte cette évolution. Désormais, si les femmes restent très présentes au Salon, elles se cantonnent à des sujets plus conformes à la prétendue tradition de leur sexe, natures mortes ou scènes d'histoire à la "sentimentalité exacerbée ${ }^{78}$ ».

De même, force est de constater que les femmes brident désormais leur ambition dans le domaine des autoportraits. Pour la plupart, ceux-ci ne représentent plus l'artiste mais la femme. De la Restauration à la fin de la monarchie de Juillet, les autoportraits individuels de femmes en tant que peintres ne sont plus qu'épisodiques. Ils sont remplacés par des portraits de groupe, des Intérieurs d'atelier. Précisons que, sur la même période, les autoportraits individuels d'artistes hommes dans leurs ateliers 
continuent d'être régulièrement exposés au Salon. Il ne s'agit donc pas d'une tendance générale de l'École française, mais bien d'un phénomène strictement limité aux femmes.

Le Tableau de feue Madame Vincent, élève de son mari de Marie-Gabrielle Capet, évoqué plus haut, n'est qu'un signe avant-coureur. Les Intérieurs d'atelier en guise d'autoportraits, peints par des femmes, confirment et amplifient cette tendance dans les décennies suivantes. Ces artistes ne sont plus désormais que les élèves, sœurs ou compagnes, qui font fonction d'assistantes du "maitre». Adrienne Marie-Louise Grandpierre-Deverzy ${ }^{79}$ prépare les couleurs ${ }^{80}$ de son professeur et futur époux, Abel de Pujol. Léon Cogniet ${ }^{81}$ qui tient un des principaux ateliers pour jeunes filles est également célébré par sa sœur, Marie-Amélie ${ }^{82}$ qui se représente à ses côtés, derrière le chevalet $^{83}$; son élève et future épouse, Caroline Thévenin ${ }^{84}$, fait de même ${ }^{85}$ :

«L'impression première devant cette toile est celle d'une réunion de jeunes filles de bonnes familles passant du temps dans un atelier d'artiste. L'idée de passe-temps l'emporte sur celui de profession. Pas de modèles ici, posant ou au repos, mais un enseignement basé sur la copie ${ }^{86}$.

Ces représentations d'artistes femmes dans le cadre d'Intérieurs d'atelier sont enfin progressivement abandonnées dans les premières années de la monarchie de Juillet. Au Salon de 1836, Adrienne Marie-Louise Grandpierre-Deverzy représente toujours l'Intérieur d'atelier de M. A. P., Peintre d'histoire ${ }^{87}$, mais elle n'y figure même plus.

Désormais, les femmes sont certes présentes en nombre, mais ne se revendiquent plus explicitement en tant qu'artistes. Leurs autoportraits sont plus rares; ce sont souvent des pastels ou des miniatures, et certains tableaux sont particulièrement conventionnels, tel l'Autoportrait de Jeanne Belloc ${ }^{88}$ en costume de novice de la maison royale de la Légion d'honneur ${ }^{89}$. Seul un autoportrait fait figure d'exception : celui d'Hortense Haudebourt-Lescot ${ }^{90}$ en $1825^{91}$, où l'artiste se réfère tant à Rembrandt par le grand bonnet noir qu'au Balthazar Castiglione de Raphaël par la pose.

\section{L'effacement relatif des femmes peintres se prolonge jusqu'au dernier quart du XIX ${ }^{e}$ siècle}

43 Le terme de « parenthèse enchantée » utilisé par Séverine Sofio pour qualifier la fin du $\mathrm{xVIII}^{\mathrm{e}}$ siècle pour les femmes dans le monde artistique mérite sans doute d'être relativisé, le statut des femmes, et en particulier des artistes, restant alors l'objet d'un combat permanent; toutefois, cette période a véritablement été marquée par une éphémère avancée de la présence des femmes au sein du Salon, et leurs autoportraits attestent qu'elles se sont alors effectivement représentées en tant que peintres, à l'égal de leurs confrères. Au tournant du siècle leur espace de liberté se restreint. La pression sociale les enferme dans l'étau des conventions bourgeoises du xix siècle. L'ordre moral triomphe, et leurs autoportraits montrent non plus l'artiste, voire le professeur, mais l'épouse, ou, au mieux, la collaboratrice du peintre. Dans la seconde moitié du siècle, la pression se relâche. Les normes collectives s'effritent et, comme le soulignent Geneviève Fraisse et Michèle Perrot, les limites apposées à la vie civile féminine disparaissent progressivement ${ }^{92}$. Parallèlement, quelques femmes arrivent au premier plan avec, par exemple, la peintre animalière Rosa Bonheur, puis les impressionnistes Berthe Morisot, Marie Bracquemond ou l'américaine Mary Cassatt. 


\section{Un épilogue révélateur : le Louvre refuse le legs de l'Autoportrait avec deux élèves}

Ce désintérêt vis-à-vis des femmes peintres a sans doute contribué au rejet par le musée du legs de l'Autoportrait avec deux élèves de Labille-Guiard. Les faits sont clairs : dans un codicille à son testament, Madame Auguste-François Briois, veuve d'un descendant de François-André Vincent, lègue le tableau au Louvre, sous réserve d'usufruit au profit de son fils Auguste Griois. Le 9 mai 1878, moins de trois mois après le décès de sa mère, ce dernier écrit à Eugène Guillaume, Directeur général des BeauxArts, contestant implicitement le droit de sa mère à disposer de ce tableau, en prétextant, au mépris des faits, que l'Autoportrait avec deux élèves était « un portrait de ma tante, fait pour elle alors que Vincent, mon grand-oncle, était Directeur de l'École de Rome et pour son retour, voilà tout. » Six mois plus tard, Eugène Guillaume explique au Ministre de l'Instruction publique, son autorité de tutelle, que M. Le Directeur des Musées nationaux, consulté, estime que « ces toiles sans valeur artistique trouveraient difficilement place dans nos collections nationales »; le Directeur des Beaux-Arts ajoute que M. Griois regretterait de devoir se séparer d'un « souvenir de famille ». Conclusion : le musée refuse le legs le 4 décembre $1878^{93}$.

«Souvenir de famille » ou non, la veuve d'Auguste Griois vendra le tableau en 1905. Il sera finalement légué en 1953, sous réserve d'usufruit, au Metropolitan Museum qui, lui, ne refusera pas le legs, et entrera en 1961 dans les collections du musée newyorkais. On a pu l'admirer à Paris en 2015, à l'occasion de l'exposition Vigée Le Brun au Grand Palais.

\section{NOTES}

1. L'existence de ce vocable est documentée plus tôt dans les pays anglo-saxons. L'Oxford English Dictionnary date l'apparition du terme self-portrait de 1831, alors qu'en allemand, les termes Selbstbildniss et Selbstporträt sont introduits dans les années 1830-1840. Cependant, tant en Angleterre qu'en Allemagne, l'utilisation du mot «autoportrait » ne commence à s'étendre que dans le dernier quart du XIX ${ }^{\mathrm{e}}$ siècle.

2. Voir l'ouvrage séminal de Pascal Bonafoux, Les Peintres et l'autoportrait, Genève, Skira, 1984.

3. Omar Calabrese, L'art de l'autoportrait. Histoire et théorie d'un genre pictural, trad. Odile Ménégaux et Reto Morgenthaler, Citadelles \& Mazenod, Paris, 2006.

4. Philippe Lejeune, « Regarder un autoportrait », Corps écrit, 5/1983.

5. La dernière en date étant Figure d'artiste au musée du Louvre, Petite Galerie (septembre 2019juin 2021).

6. Dans les écrits récents, deux séries de termes sont utilisés, d'une part «femmes peintres ou «femmes artistes », d'autre part " peintres femmes» ou «artistes femmes». Les tenants de la première formulation insistent sur la discrimination subie par les femmes dans le monde artistique et leur lutte en faveur d'une égalité de traitement; voir notamment Marie-Josèphe Bonnet, Liberté, égalité, exclusion, Femmes peintres en Révolution, 1770-1804, Paris, Vendémiaire, 2012, M.-J. Bonnet, «Femmes-peintres à leur travail: de l'autoportrait comme manifeste politique 
(XVIII ${ }^{\mathrm{e}}$ XIX ${ }^{\mathrm{e}}$ siècles) », Revue d'histoire moderne et contemporaine, 3/2002 ( ${ }^{\circ}$ 49-3) ; M.-J. Bonnet, « La Révolution d'Adélaïde Labille-Guiard et Élisabeth Vigée Le Brun, dans Les Femmes et la Révolution française, PUM, t. 2, 1988. Les partisans de la seconde formulation mettent plutôt l'accent sur l'épanouissement des femmes dans et par l'art à la fin du XVIII ${ }^{\mathrm{e}}$ siècle ; voir à ce sujet Séverine Sofio, Artistes femmes, La parenthèse enchantée, XVIII $I^{e}-X_{X}{ }^{e}$ siècles, Paris, CNRS, coll. "Culture \& société », 2016. Dans un souci d'harmonisation des termes, et sans nous prononcer pour autant sur le fond du débat suscité par l'appellation «femmes peintres », qui sous-entendrait «femme avant que d'être peintre »- Martine Lacas (sous la dir. de), Peintres Femmes, Naissance d'un combat, 1780-1830, cat. d'exp., Paris/musée du Luxembourg (mars-juillet 2021), Paris, éditions de la RMN, 2021 , p. 25 -, nous utiliserons ici les expressions «femmes peintres » et femmes artistes ", dans la mesure où, dans le cadre historique concerné, l'enjeu est bien la reconnaissance des femmes en tant qu'artistes à part entière.

7. Voir note 6.

8. Frances Borzello, Femmes au miroir, une histoire de l'autoportrait féminin, trad. Marie Muraccioli, Londres-Paris, Thames and Hudson, 1998.

9. Cette exposition, initialement prévue de septembre 2020 à janvier 2021, et reportée en raison de la crise sanitaire liée à la COVID-19 à mars-juillet 2021, devrait ouvrir le 19 mai 2021. Il a été néanmoins possible de faire une visite virtuelle de l'exposition avant l'ouverture, grâce au site du musée https://museeduluxembourg.fr/fr/actualite/visitez-lexpo-peintres-femmes-depuis-chezvous (dernière consultation le 6 mai 2021).

10. Voir note 6.

11. M. Lacas, op. cit. note 6, p. 19.

12. Rosalie Filleul de Besnes, née Boquet (1752-1794), fille du peintre ornemaniste Blaise Boquet (vers 1721-1784), peintre et pastelliste, a été admise à l'Académie de Saint-Luc en 1773. Elle se fait remarquer par ses portraits à la dernière exposition de l'Académie de Saint-Luc, en 1774, mais ralentit son activité de portraitiste après son mariage en 1777. Rosalie Filleul n'émigre pas et est guillotinée en 1794, en compagnie d'une de ses amies, Marguerite Émilie Chalgrin (1760-1794), fille du peintre Joseph Vernet (1714-1789) et épouse de l'architecte Jean-François Chalgrin (1739-1811). L'Autoportrait de Rosalie Filleul présenté à l'exposition du musée du Luxembourg est en mains privées.

13. Voir Melissa Hyde, "Espaces et stratégies des peintres femmes ", dans M. Lacas (sous la dir. de), Peintres Femmes, Naissance d'un combat, op. cit. note 6, p. 91.

14. Julie Duvidal de Montferrier (1797-1865), élève du Baron Gérard, participe aux Salons de 1819, 1822 et 1824 . Elle épouse en 1827 le frère de Victor Hugo et est connue également comme la comtesse Abel Hugo. Gérard fera le portrait de son ancienne élève en 1830 sous le nom de Portrait de Julie Hugo. Ce tableau est conservé à la maison de Victor Hugo, Hauteville House, à Guernesey (INV 264). L'Autoportrait de Julie Duvidal de Montferrier a été présenté au Salon ( $\mathrm{N}^{\circ} 1631$ ) avec son pendant (le portrait de la sœur de l'artiste, Zoé) sous le titre Portrait de Melles ***. Il est conservé à l'École nationale des Beaux-Arts (INV MU 10800). Ni le mode d'acquisition ni la date ne sont mentionnés. Voir également Monique Moulin, «Une élève de Gérard: Julie Duvidal de Montferrier ", Bulletin de la Société historique de Compiègne, tome 27, 1980, pp. 169-181.

15. Udolpho Van de Sandt, «La fréquentation des Salons sous l'Ancien Régime, la Révolution et l'Empire ", Revue de l'Art 3, 1986, pp. 43-48 et U. Van de Sandt, Histoire des expositions de l'Académie royale de peinture et de sculpture (1663-1791), Solennité, Fêtes, Cérémonies, Concours et Salons, Paris, DL, 2018.

16. Voir Pascal Griener, Paul-André Jaccard, Paris! Paris! Les artistes suisses à l'École des Beaux-Arts (1792-1863), Paris, Slatkine, 2014.

17. Cette recherche a été effectuée dans le cadre de la thèse de troisième cycle en cours à l'École du Louvre, sous la direction de Cecilia Hurley et Vincent Delieuvin, Les autoportraits au Louvre, hasard et nécessité. 
18. Les livrets des Salons des $\mathrm{XVII}^{\mathrm{e}}$ et $\mathrm{XVIII}{ }^{\mathrm{e}}$ siècles ont été publiés en neuf volumes entre 1869 et 1872 par Jules Guiffrey sous le titre Collection des livrets des anciennes expositions depuis 1673 jusqu'en 1800, et réédités par Jacques Laget, Nogent-le-Roi, Jacques Laget, Librairie des Arts-et-Métiers Éditions, 1990-1991. Par ailleurs, depuis le milieu des années 2010, ces livrets ont été mis en ligne par le musée d'Orsay, voir http://salons.musee-orsay.fr/.

19. Adélaïde Labille-Guiard (1749-1803), élève du miniaturiste François-Élie Vincent, étudie le pastel avec Quentin de La Tour. Reçue à l'Académie comme portraitiste en 1783, la même année qu'Élisabeth Vigée Le Brun, l'artiste présente une quinzaine de portraits au Salon de 1783, et huit à celui de 1785 , dont L'Autoportrait avec deux élèves $\left(\mathrm{N}^{\circ} 101\right)$. Nommée Premier Peintre de Mesdames en 1787, Labille-Guiard s'adapte au nouveau contexte politique au début de la Révolution en faisant le portrait de plusieurs conventionnels dont Robespierre, mais se réfugie à la campagne pendant la Terreur. L'artiste est de nouveau présente au Salon après Thermidor, de 1795 à 1800, année où elle expose sous le nom de « Mme Vincent (ci-devant Guiard) », après avoir épousé en secondes noces le peintre François-André Vincent.

20. Nous savons que nous sommes ici en contradiction avec Martine Lacas, qui a récemment contesté tout " cadre interprétatif fondé sur l'hypothèse d'un progrès ou d'une décadence » (voir M. Lacas, op. cit. note 6, p. 20). Si le terme « décadence » utilisé dans le titre peut sembler fort, il est utilisé à dessein, car il n'en décrit pas moins une réalité, comme l'examen des autoportraits de femmes peintres de la première moitié du XIX ${ }^{\mathrm{e}}$ siècle va le montrer.

21. Marie-Marguerite Carrreaux de Rosemond (1765-1788) et Marie-Gabrielle Capet (1761-1818). Cette dernière restera très proche d'Adélaïde Labille-Guiard et habitera avec elle et son époux jusqu'au décès de celle-ci.

22. Mouffle d'Angerville, dans Louis Petit de Bachaumont, Les salons de Bachaumont, Chroniques esthétiques du XVIII ${ }^{e}$ siècle, Introduction et analyse par Fabrice Faré, Jacques Laget, 1995, Salon de 1785, Lettre II, p. 131.

23. On peut ainsi lire dans un pamphlet:

"A Madame Guiard

Que vois-je, ô ciel l'ami Vincent

Ne va donc plus que d'une fesse.

Son amour fait votre talent.

L'amour meurt, le talent baisse, bis

Résignez-vous, bis, fière Cloris,

Dites votre De Profundis »

Anonyme, Suite de "Marlborough au Salon $1783 »$. Confession promise par le peintre allemand, 1783, Coll. Deloynes, t. 13 , pièce 302.

24. François-André Vincent (1746-1816), fils du miniaturiste François-Élie Vincent (1708-1790) originaire de Genève, étudie la peinture avec son père avant d'être l'élève de Joseph Marie Vien (1716-1809) et d'être reçu à l'Académie comme peintre d'histoire en 1782. Initiateur du courant néoclassique, Vincent est le rival de David, qui finira par le supplanter peu avant la Révolution. À partir de 1792, Vincent se consacre de plus en plus à l'enseignement, notamment à l'École Polytechnique. Il épouse Adélaïde Labille-Guiard en 1799.

25. Comme le rappelle Octave Fidière : «pour faire tomber le préjugé qui s'attache quelquefois aux œuvres sorties de la main d'une femme, elle eut l'idée ingénieuse de faire les portraits de membres de l'Académie, afin qu'ils sussent par eux-mêmes si tout son talent lui appartenait. » Octave Fidière, Les femmes artistes à l'Académie royale de peinture et de sculpture, Paris, Charavay, 1885 , p. 10.

26. Stéphane Guégand, «Une femme de lumière », dans Joseph Baillo, Xavier Salmon (sous la dir. de), Élisabeth Louise Vigée Le Brun, cat. d'exp., Paris/Grand Palais (septembre 2015-janvier 2016), New York/Metropolitan Museum (février-mai 2016), Ottawa/Musée des Beaux-Arts du Canada (juin-septembre 2016), Paris, RMN, 2015, p. 61. 
27. L'Académie de Saint-Luc, qui regroupe peintres, sculpteurs et graveurs et accueille des femmes, en nombre certes infime, environ $3 \%$ (cent trente femmes sur quatre mille cinq cent maitres entre 1617 et sa dissolution), est moins prestigieuse que le Salon, mais organise de manière épisodique des expositions pour ses membres. Entre 1751 et 1774, on compte sept expositions. L'Académie de Saint-Luc disparaît en 1777, en application de l'édit du roi supprimant les jurandes, dit édit de Turgot, de février 1776. Voir à ce sujet Ann Sutherland Harris et Linda Nochlin (sous la dir. de), Femmes peintres : 1550-1950, cat. d'exp., Los Angeles/Los Angeles County Museum of Art (1976-1977) ; Austin/University Art Museum (1977) ; Pittsburg/Carnegie Museum of Art (1977), trad. par Claude Bourguignon, Pascale Germain, Julie Pavesi et Florence Verne, Paris, des femmes, 1981, p. 37.

28. Ci-après dénommée simplement Académie.

29. André Félibien (1619-1695), historien et critique d'art. Secrétaire d'ambassade à Rome, il se lie avec Poussin. Revenu en France, il dédicacera son ouvrage sur L'Origine de la peinture, fondée sur les écrits de Giorgio Vasari, à Nicolas Fouquet, alors surintendant des Finances. Après une brève disgrâce, liée à celle de son protecteur, Félibien est nommé par Colbert historiographe des Bâtiments du roi en 1666. Considéré comme un des principaux théoriciens du classicisme, il publie une histoire de la peinture, les Entretiens sur les vies et les ouvrages principaux des plus excellents peintres anciens et modernes (1666-1688). Félibien liste des genres par ordre d'importance : au sommet il y a l'histoire, le grand genre ou genre noble, qui traite de l'histoire sainte, antique, médiévale ou moderne et de l'allégorie. En deçà, on trouve le portrait, la scène de genre, la nature morte et le paysage.

30. Catherine Duchemin (1630-1698), épouse du sculpteur François Girardon (1628-1715).

31. Anatole Montaiglon, Procès-verbaux de l'Académie royale de peinture et de sculpture, 1648-1793, t. I, 1648-1672, Paris, J. Baur libraire, 1875, p. 223.

32. Geneviève (1645-1708) et Madeleine Boullogne (1648-1710), filles du peintre Louis Boullogne (1609-1674), avaient été admises comme peintres de fleurs.

33. A. Montaiglon, op. cit. note 20, t. IV, pp. 34-35.

34. L'Italienne Rosalba Carriera (1675-1737) est admise par acclamation en octobre 1720. Marguerite Haverman (active au XVIII ${ }^{\mathrm{e}} \mathrm{s}$ ), peintre d'origine hollandaise est reçue en 1722 avant d'être rayée des registres l'année suivante, faute d'avoir présenté son morceau de réception. Étant étrangères, elles ne créent pas de précédent dont puissent se prévaloir des artistes françaises.

35. Mammès-Claude Pahin Champlain de La Blancherie (1752-1811) suit une formation juridique avant de se tourner vers le journalisme et de fonder les Nouvelles de la république des Lettres et des Arts, qui est l'organe du Salon de la Correspondance. En 1788, l'échec de cette entreprise, faute de soutien de la part des pouvoirs publics, entraine le départ de La Blancherie pour l'Angleterre, où il se consacre à la célébration d'Isaac Newton et publie notamment un «Plan à la mémoire de Newton ». Il passe la fin de sa vie de l'autre côté de la Manche.

36. Thomas Crow, La Peinture et son public à Paris au XVIII ${ }^{e}$ siècle, trad. A. Jacqueson, Yale, 1985, Paris, Éditions Macula, 2000 pour la version française, p. 97.

37. Pour le Salon de la Correspondance et l'Exposition de la jeunesse, consulter notamment: «Exposition de tableaux à la place Dauphine le jour de la petite fête Dieu tirée de l'AvantCoureur ", 1760, Coll. Deloynes, t. 48, pièces 1268 à 1296, Supplément au T. III, pp.3-12; «Exposition de tableaux a la place Dauphine tirée de l'Avant-Coureur », 1761, Coll. Deloynes, t. 48, pièces 1268 à 1296, Supplément au T. III, pp. 239-246; "Exposition de tableaux à la place Dauphine ", Avant-Coureur, 1767, Coll. Deloynes, t. 49, pièces 1297 à 1335, Supplément au T. IV, pp. 267-270.

38. Anne-Dorothée Therbusch, née Liszewska (1721-1782), d'origine polonaise mais née en Prusse, a été admise en 1767, mais l'artiste a quitté la France avant même le Salon où elle avait envoyé plusieurs portraits. Voir O. Fidière, op. cit. note 15, pp. 39-40. 
39. Anne Vallayer-Coster (1744-1818), est admise à l'Académie en 1770, et expose quinze fois au Salon entre 1771 et 1817 . Les deux tableaux présentés en 1770 lui servent de morceau de réception, en tant que "peintre de natures morte". Active sous son nom de jeune fille à ses débuts, Anne Vallayer fait adjoindre le nom de son époux à partir de 1781. Elle expose quinze fois au Salon entre 1771 et 1810 , surtout des natures mortes et quelques portraits.

40. Moins d'un mois plus tard «la Dame Marie-Suzanne Giroust, épouse de M. Roslin, Conseiller [...], Peintre dans le genre du Portraict [...] en Pastel » est également agréée et reçue le même jour. A. Montaiglon, op. cit. note 20, t. IV, p. 52.

41. L'orthographe des citations a été respecté, hormis les terminaisons des verbes en « oit » qui ont été transcrites en " ait ».

42. A. Montaiglon, op. cit. note 20, t. IV, p. 53.

43. Par ordre chronologique : Catherine Duchemin ; Geneviève et Madeleine Boullogne ; Élisabeth Sophie Chéron; Anne Renée Strésor; Dorothée Massé; Catherine Perrot; Rosalba Carriera; Margareta Haverman; Marie-Thérèse Reboul, épouse du peintre Marie-Joseph Vien; AnneDorothée Therbusch, née Liszewska ; Anne Vallayer-Coster; Marie-Suzanne Giroust, épouse du peintre Roslin ; Adélaïde Labille-Guiard et Élisabeth Vigée Le Brun.

44. Marguerite Gérard (1761-1837), élève de Fragonard (1732-1806), époux de sa sœur MarieAnne, est une des femmes artistes les plus importantes de l'extrême fin du xvIII siècle et du premier tiers du XIX ${ }^{\mathrm{e}}$, appréciée comme peintre de scènes de genre dans le style de la peinture hollandaise $\mathrm{du} \mathrm{XVII}{ }^{\mathrm{e}}$ siècle et de petits portraits familiers. Elle réalise également des portraits de personnalités contemporaines, comme le Portrait de Madame de Staël et de sa fille Albertine (Château de Coppet) ou le Portrait de la duchesse d'Abrantès et du général Junot (coll. part.), alors que sa Clémence de Napoléon (Château de La Malmaison) est achetée par l'Empereur en 1808.

45. Voir Carole Blumenfeld, Marguerite Gérard, 1761-1837, Montreuil, Éd. Gourcuff Gradenigo, 2019, p. 23. C'est indirectement grâce à la présence d'œuvres de Marguerite Gérard chez de nombreux collectionneurs que le musée de l'Ermitage de Saint-Pétersbourg bénéficie de trois tableaux, Une artiste faisant le portrait d'une musicienne (гэ-1129), Le Présent (гэ-6649) et Les Premiers pas (гэ-1129), tandis que le Musée Pouchkine à Moscou abrite La Maternité (Ж-919).

46. Ce tableau (RF2019.51.1) a été acquis par exercice du droit de préemption de l'État lors de la vente de la collection du comte et de la comtesse de Ribes, organisée par Sotheby's en décembre 2019.

47. Ce tableau sera exposé au Salon de 1783 , sous le $\mathrm{N}^{\circ} 119$.

48. M.-J. Bonnet, Liberté, égalité, exclusion, Femmes peintres en Révolution, 1770-1804, op. cit. note 6, p. 37.

49. Anonyme, Lettre à MM. les rédacteurs du Mercure de France, 1786, coll. Deloynes 360, pp. 883-884.

50. L. P. de Bachaumont, op. cit. note 12, p. 109.

51. Ibid., p. 118.

52. Une réplique autographe de L'Autoportrait au chapeau de paille d'Elisabeth Vigée Le Brun est conservée à la National Gallery de Londres (NG 1683), l'original étant dans une collection particulière. Le tableau d'Adélaïde Labille-Guiard, pastel de forme ovale, légué en 1816 par François-André Vincent à M ${ }^{\text {elle }}$ Victoire d'Avril, une des élèves de l'artiste, n'est pas localisé.

53. Joanna Woods-Marsden, Renaissance Self-Portrait : the visual construction of identity and the social status of the artist (1936), New Haven, Yale University Press, 1998, p. 5.

54. Décret de l'Assemblée constituante du 21 août 1791, article I ${ }^{\mathrm{er}}$.

55. La tendance se poursuivra après la date limite de notre champ d'étude, et le nombre de femmes exposant au Salon atteindra le chiffre record de 844 en 1879.

56. Voir M.-J. Bonnet, Liberté, égalité, exclusion, Femmes peintres en Révolution, 1770-1804, op.cit. note 6, p. 140 
57. Ces sculptures seraient d'une part le portrait du père d'Adélaïde, Claude-Edme Labille, par Pajou, d'autre part une Vestale de Jean-Antoine Houdon. Voir Laura Auricchio, Adélaïde LabilleGuiard. Artist in the Age of Revolution, Los Angeles, The Paul Getty Museum, 2009, p. 47.

58. Louise-Élisabeth Vigée Le Brun, Souvenirs 1755-1842, texte établi et annoté par Geneviève Haroche-Bouzinac, Paris, Honoré Champion, 2015, Lettre VI, pp. 179-180.

59. Comte Stanislas Kostka Potocki (1755-1821), homme politique, écrivain et mécène.

60. Cité par Jean-Pierre Cuzin, « Hommage à Raphaël », Raphaël et l'art français, cat. d'exp., Paris/ Grand Palais, novembre 1983-février 1984, Paris, Éd. de la RMN, 1983, p. 178.

61. Constance Mayer (1775-1821), peintre de genre, pastelliste et miniaturiste, est l'élève, la collaboratrice et la compagne de Pierre-Paul Prud'hon (1758-1823).

62. Salon de 1801, № 238.

63. Marie Suzanne Roslin, née Giroust (1734-1772), élève de Vien et d'Alexandre Roslin qu'elle épouse, est reçue à l'Académie en 1770 comme pastelliste.

64. Marie-Guilhelmine Benoist, née de Laville-Leroulx (1768-1826), portraitiste, expose régulièrement au Salon entre 1795 et 1812. Son Portrait d'une négresse [sic] a créé l'évènement au Salon de 1800. Le modèle a été identifié et l'œuvre, conservée au Louvre (INV 2508), porte aujourd'hui le nom de Portrait de Madeleine.

65. Jean-Baptiste Jacques Augustin (1759-1832), spécialisé dans l'art de la miniature, reçoit en 1824 le titre de Premier peintre en miniature du cabinet du roi. Plusieurs de ses Autoportraits sont conservés au département des Arts graphiques du Louvre.

66. Jean Siméon Chardin (1699-1779), peintre de genre, de portraits et de natures mortes, reçu à l'Académie en 1727, s'est tourné vers le pastel en raison de ses problèmes de vue à la fin de sa vie. Il présente, sous le titre générique de Têtes d'étude, un autoportrait lors de trois Salons: l'Autoportrait aux besicles (1771), l'Autoportrait à l'abat-jour (1775) et enfin l'Autoportrait au chevalet (1779). Ces trois pastels sont conservés au Louvre (INV 2506, INV 2507 et RF 31748).

67. Joseph Ducreux (1735-1802), portraitiste. Premier peintre de la Reine, il ne peut se faire accepter à l'Académie, ayant dit trop de mal de cette institution, et doit donc attendre 1791 pour exposer au Salon. Auteur de nombreux autoportraits, le peintre explore l'éventail des expressions, tantôt baillant, tantôt surpris, ou ricanant.

68. Louis-Michel Van Loo (1707-1771) est un des derniers représentants d'une dynastie de peintres. Peintre officiel du roi Philippe V d'Espagne de 1736 à 1752, il s'illustre par des portraits tant de la Cour d'Espagne que de la Cour de France. Le tableau est conservé au château de Versailles (MV 6774).

69. Simon-Charles Miger (1736-1820) se consacre à la gravure après avoir été formé par CharlesNicolas Cochin. Admis à l'Académie en 1781, Miger est surtout connu pour avoir gravé les planches de La Ménagerie du Museum d'histoire naturelle, ou les animaux vivants, d'après les aquarelles de Nicolas Maréchal au tout début du XIX ${ }^{\mathrm{e}}$ siècle.

70. Jean-Michel Moreau (1741-1814), dit Moreau le Jeune, est le frère cadet du peintre et graveur à l'eau-forte Louis-Gabriel Moreau (1740-1806), dit l'Aîné. Dessinateur, peintre et graveur, Moreau le Jeune est nommé en 1770 dessinateur des Menus Plaisirs du Roi, puis en 1781 dessinateur et graveur du Cabinet du Roi. Agréé à l'Académie royale de peinture et de sculpture en 1780 , puis reçu membre en 1788 , il expose régulièrement au Salon entre 1781 et 1810 . Artiste prolifique, son œuvre est riche de plus de 2000 dessins dont la plupart furent gravés par luimême ou d'autres artistes.

71. À cette époque, sur les quatre femmes Académiciennes, seules deux sont actives dans cette enceinte: Marie-Thérèse Vien, à la santé fragile, ne participe plus aux réunions, et Élisabeth Vigée Le Brun est en exil. Pour sa part, Anne Vallayer-Coster ne fait que signer une pétition contre les réformateurs. S. Sofio, op. cit. note 6, p. 114.

72. A. Sutherland Harris, op. cit. note 17, p. 37. 
73. Jacques-Louis David (1748-1825) est reçu à l'Académie en 1783 comme peintre d'histoire et s'impose comme chef de file du néoclassicisme. Député de la Convention, membre du Comité de sûreté générale et organisateur des célébrations révolutionnaires, David, accusé de trahison après la chute de Robespierre, est arrêté et reste sept mois en prison, avant d'être relâché. Admirateur de Bonaparte, il devient Premier peintre de l'Empereur. À la chute de l'Empire, David s'exile à Bruxelles, où il termine ses jours.

74. L. Nochlin, op. cit. note 17, p. 45.

75. Seules, les veuves ont, en théorie, la capacité juridique, mais si elles ont des enfants elles sont étroitement encadrées par un Conseil de famille dont les membres sont issus de la famille de leur conjoint décédé.

76. Article 213 du Code civil.

77. Geneviève Fraisse, Michèle Perrot, «Ordre et liberté », Histoire des femmes en Occident, t. IV, Le XIX ${ }^{e}$ siècle, Paris, Plon, 2002, p. 14.

78. S. Sofio, «La vocation comme subversion, Artistes-femmes et anti-académisme dans la France révolutionnaire », Actes de la recherche en sciences sociales 2007/3 ( $\left.\mathrm{N}^{\circ} 168\right)$, p. 28.

79. Adrienne Marie-Louise Grandpierre-Deverzy (1798-1865), élève, puis assistante et enfin épouse d'Abel de Pujol, réalise de nombreux portraits et des scènes de genre dans le style troubadour.

80. Salon de $1822, \mathrm{~N}^{\mathrm{o}} 605$.

81. Léon Cogniet (1794-1880), peintre d'histoire et portraitiste, se consacre à l'enseignement à partir de 1843. Il tient en fait deux ateliers séparés, l'un pour jeunes hommes, l'autre pour jeunes filles. Celui-ci est sous la responsabilité directe de sa sœur: "C'est elle qui encadre l'enseignement de l'atelier féminin, comme il était de coutume, les peintres délaissant à d'anciennes élèves la charge de suivre à la semaine l'atelier, ces derniers ne venant que ponctuellement corriger et conseiller les travaux des jeunes filles. La deuxième femme à avoir secondé Léon Cogniet dans la mise en place d'un atelier pour jeunes filles est une de ses élèves : Caroline Thévenin (1813-1892), qui devient sa femme en 1865. » Voir Michael Vottero, « Le cri de la conscience : Léon Cogniet et ses ateliers », Territoires contemporains 4, Image de l'artiste,

http://tristan.u-bourgogne.fr/CGC/publications/image_artiste/Michael_Vottero.html [consulté le 2 février 2020)].

82. Salon de 1831, № 351.

83. Marie-Amélie Cogniet (1798-1869), qui expose régulièrement au Salon de 1831 à 1843, représente l'atelier de son frère et professeur, Léon Cogniet (1794-1880) dans une série de petits formats, dont l'un est présenté au Salon de 1831 ( $\left.\mathrm{N}^{\circ} 361\right)$.

84. Catherine Caroline Thévenin (1813-1892), élève puis épouse de Léon Cogniet.

85. Salon de $1836, \mathrm{~N}^{\mathrm{o}} 1727$.

86. M. Vottero, op. cit. note 67.

87. No 890.

88. Jeanne Belloc (1811-1889), fille et élève du peintre d'histoire et portraitiste Jean-Hilaire Belloc (1786-1856), expose au Salon de 1835 à 1868.

89. Salon de $1836, \mathrm{~N}^{\circ} 120$.

90. Hortense Haudebourt-Lescot (1784-1845), peintre d'histoire et de portraits.

91. Le tableau est entré en 1867 dans les collections du Louvre (MI 719).

92. G. Fraisse, M. Perrot, op. cit. note 62, p. 14.

93. Voir AN 20144790/70 pour l'ensemble de la correspondance. 


\section{RÉSUMÉS}

À partir d'une étude quantitative et qualitative, cet article a pour objectif de montrer l'importance de l'autoportrait pour les artistes femmes, véritable passeport pour le Salon au XVIII . À l'orée de la Révolution, l'autoportrait «à la peinture » est pour les femmes le véhicule d'une véritable revendication, artistique mais aussi politique. Le contexte socio-économique change au début du XIX ${ }^{\mathrm{e}}$, avant de constater l'effacement progressif de leur rôle, mis en évidence par une transformation radicale de la manière dont elles se représentent. Au XIX ${ }^{\mathrm{e}}$, les valeurs bourgeoises enferment la femme, dont la seule vocation devient la maternité. A vingt-trois ans de distance, deux représentations d'Adélaïde Labille-Guiard, - son Autoportrait avec deux élèves de 1785 et le Tableau représentant feue Madame Vincent, élève de son mari de Marie-Gabrielle Capet résument cette évolution. Désormais, les femmes brident désormais leur ambition dans le domaine des autoportraits. Pour la plupart, ils ne représentent plus l'artiste mais la femme.

Based on a quantitative and qualitative study, this article aims to demonstrate the importance of the self-portrait to woman artists, which was a passport to the Salon in the eighteenth century, then observe the gradual diminution of their role, highlighted by a radical transformation in the way they represented themselves. For women on the eve of the French Revolution, the selfportrait "à la peinture" was a genuine artistic and political statement. The socioeconomic context changed in the early nineteenth century as bourgeois values confined women, whose only vocation became motherhood. Two representations of Adélaïde Labille-Guiard, twenty-three years apart - her Self-Portrait with Two Pupils from 1785 and the Painting Representing the Late Madame Vincent, Pupil of Her Husband by Marie-Gabrielle Capet - sum up this change. Henceforth, women limited their ambitions to the field of self-portraits. For the most part, they no longer represented the artist, just the woman.

\section{INDEX}

Index chronologique : XVIIIe siècle, XIXe siècle

Keywords : Self-portrait, woman artist, woman painter, Salon, Académie Royale de Peinture, Académie Saint-Luc, France, eighteenth century, nineteenth century

Mots-clés : Autoportrait, artiste femme, femme peintre, Salon, Académie royale de peinture, Académie Saint-Luc, France

\section{AUTEUR}

\section{VERA DE LADOUCETTE}

Vera de Ladoucette est diplômée de l'Institut d'Etudes Politiques de Paris et d'une Maîtrise en droit public (Paris, Assas). Après une carrière dans l'énergie, elle a consacré ces dix dernières années à l'histoire de l'art. À l'Ecole du Louvre, elle a obtenu un diplôme de $1^{\text {er }}$ cycle en 2012, un Master 1 en 2013 et un Master 2 de Muséologie en 2015. Doctorante à l'École du Louvre depuis 2016 sous la direction de Cecilia Hurley-Griener et Vincent Delieuvin, elle travaille sur les autoportraits du musée du Louvre.

Vera de Ladoucette is a graduate of the Institut d'Études Politiques de Paris and has a master's degree in public law (Paris, Assas). After working in the energy sector, she has dedicated the last ten years of her life to art history. At the École du Louvre, she obtained a first degree in 2012, a 
Master 1 in 2013 and a Master 2 in Museology in 2015. A doctoral student at the École du Louvre since 2016 under the supervision of Cecilia Hurley-Griener and Vincent Delieuvin, she is writing about the self-portraits in the Musée du Louvre. 